\title{
S-CURVE ALGORITHM OF ACCELERATION/DECELERATION WITH SMOOTHLY- LIMITED JERK IN HIGH-SPEED EQUIPMENT CONTROL TASKS
}

\author{
V. Kombarov ${ }^{1 *}$, V. Sorokin¹, O. Fojtů ${ }^{1}$, Ye. Aksonov ${ }^{1}$, Ye. Kryzhyvets ${ }^{1}$ \\ ${ }^{1}$ National Aerospace University "Kharkiv Aviation Institute", Kharkiv, Ukraine \\ ${ }^{*}$ Corresponding author; e-mail: vl.kombarov@gmail.com
}

\begin{abstract}
The needs of increase in accuracy, quality and shape complexity of parts manufactured in high-speed equipment is a trend of industry. Problems of ensuring the accuracy at high velocities of machine organs motions are formulated. Machine organs movements accuracy is determined by smoothness of their motions and depends on the smoothness of the trajectory and the law of velocity changing along the trajectory. Algorithm of velocity changing along trajectory using S-curve acceleration-deceleration with smoothly-limited jerk is proposed. Experimental verification of the accuracy CNC machine organs motions at their control with using proposed S-curve algorithm showed a decrease in positioning errors.
\end{abstract}

\section{Keywords:}

CNC; S-curve algorithm of acceleration/deceleration; Smoothly-limited jerk; High-speed equipment

\section{INTRODUCTION}

One of the problem faced by equipment developers for high-speed machining is an ensuring of smooth motion of cutting tool that improves a processing efficiency, machined surface quality and tool life [Erkorkmaz 2001], [Krivtsov 2012], [Beudaert 2012]. In order to achieve high-speed and high-accuracy machining many scholars devote their investigations to parametric curve interpolation, feedrate profile scheduling and servo-loop control techniques. Investigations in the field of parametric curve interpolation and feedrate profile scheduling are aimed to formation of control by executive organs taking into account the physical phenomena occurring in an inertial system, which significantly influence a motion process. The necessity to increase feedrate with simultaneous improvement of accuracy parameters stimulates researchers to take into account the nature of differential kinematic characteristics of higher order. Earlier, at the beginning of CNC systems development the acceleration-limited models were considered and now models are being developed to ensure the acceleration changes smoothness and the jerk continuity.

[Erkorkmaz 2001] proposed the S-shape feedrate scheduling method aimed to avoiding the excitation of machine tool's structural dynamics because of high bandwidth of the drive system.

[Novikova 2012] showed that the criterion of operation smoothness for mechatronic actuators in translational motion should take into account not only the nature of change in the feedrate and acceleration time, but also in the jerk.

A large number of the models were proposed for the Sshape feedrate. For example, [Erkorkmaz 2001] used the trapezoidal acceleration profiles. Cubic polynomials for a feedrate profile with a discontinuous jerk were applied in the work [Liu 2016]. In the work [Fan 2012] a polynomial function to jerk continuous profile was applied. Some authors suggested application of trigonometric functions for the S-shape feedrate profile. The model proposed in the work [Lee 2011] provides a continuous acceleration profile with discontinuous jerk. In the work [Ni 2018] a continuous jerk profile model based on the use of sin and cos functions was proposed.

Among a large number of researches in the servo-loop control techniques, the tendency of feedforward control application with high-order differential characteristics such as acceleration [Prévost 2011] and jerk [Boerlage 2004] is observed, that provides a significant delay minimization and increase control precision for motion systems. The application of active control ensures the reduction of oscillations amplitude and frequency appeared during machining [Petrakov 2017]. The application of these control techniques needs not only the continuity, but also the smoothness of changing the movement differential characteristics such as acceleration and jerk [Krivtsov 2012], [Novikova 2012].

This paper considers a problem of determining the spline order used to describe the toolpath and suggests a model for synthesizing the high-speed machining feedrate in acceleration-deceleration process that ensures a smooth change in velocity, acceleration, jerk and continuity snap (jerk derivative).

\section{THE SMOOTHNESS HYPOTHESIS OF KINEMATIC PARAMETERS IN SPLINE INTERPOLATOR}

The main mathematical tool for modeling machining paths currently is the theory of spline functions. The B-spline [Lartigue 2001], [Zhang 2017], NURBS-splines [Lee 2011], 
[Beudaert 2012], [Liu 2016], [Ni 2018] and other types obtained the biggest application. In this paper a CNC system is considered, in which a curvilinear trajectory model is constructed in the class of fifth-degree spline functions by the method of optimal mapping into B-spline space [Sorokin 2002]. This method has high informativeness and computability of geometric and differential characteristics of motion.

Fig. 1 shows the application scheme of spline interpolation in the CNC system. Part of the interpolation tasks in the system under consideration is performed at preparation stage of the NC program: approximation, spline redefinition, velocity planning. Approximation subsystem converts a linear-circular trajectory to a spline. Spline redefinition subsystem converts spline representation. Velocity planning subsystem calculate feed rate along the path in view of specified limitations. Solving of these problems does not require real-time mode.

Feed generation and trajectory interpolation tasks are performed in the process of equipment control in real time. Feed generation subsystem calculates the feedrate value for each control cycle. Trajectory interpolation subsystem calculates the position and velocity for each axis taking into account the requirements for the order of the differential characteristics.

Modern CNC machines in terms of implementation of the tool are inertial electromechanical systems. Inertia is a resistance of any physical object to any change in its state, for example, velocity. As a result of inertia manifestation, we use a S-shaped feedrate profile with a smooth variation of velocity. Because of inertia, one cannot immediately change the forces that drive the machine elements and, as a result, we use the S-shape acceleration.

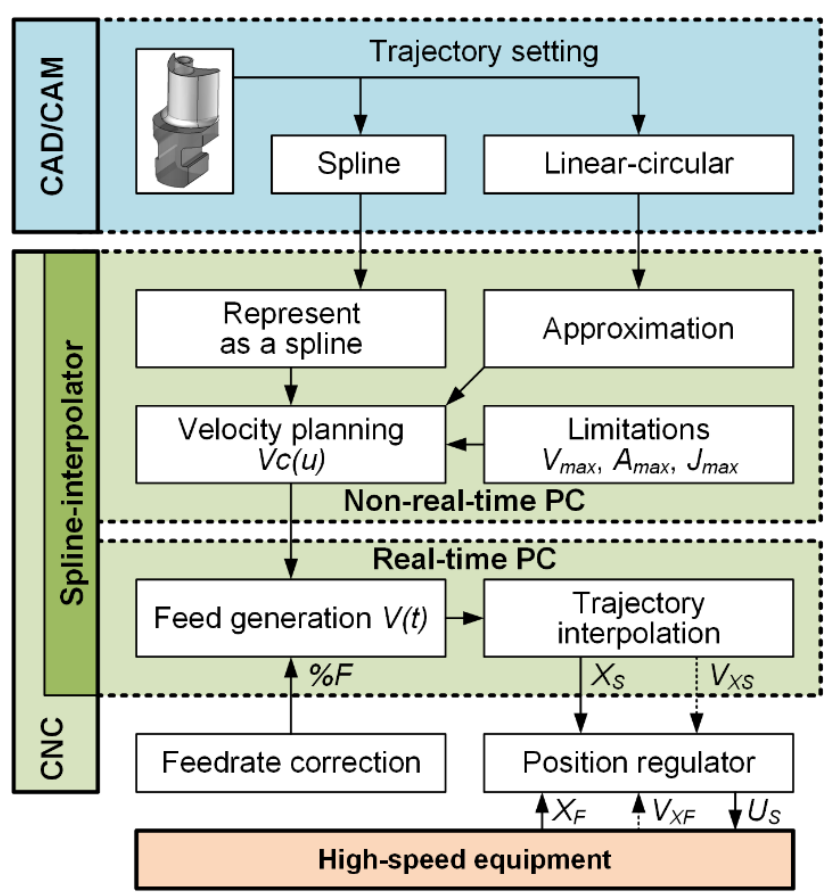

Fig. 1: Application of spline interpolation in the CNC.

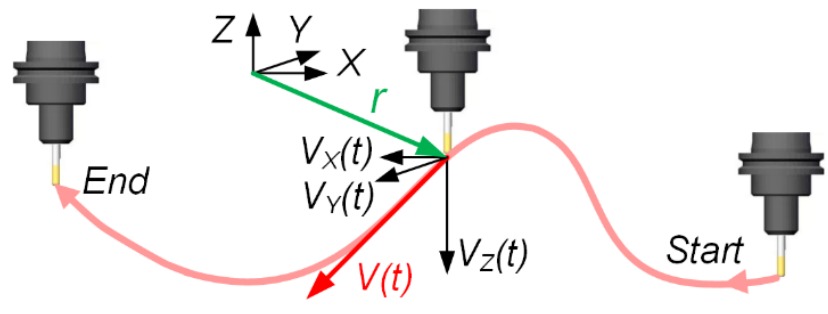

Fig. 2: Frame sample in a spline interpolator.

So, it is possible to formulate a smoothness hypothesis of kinematic parameters: if the corresponding differential characteristic has an influence on the movement process, then it should be limited and change smoothly [Krivtsov 2012], [Novikova 2012]. Respectively, if the change in the differential characteristics of a given motion is not smooth or even intermittent, it leads to deviations from a given motion profile and machining errors.

A mathematical model of a trajectory (Fig. 2) is represented as a parametric equation of a curve in a $p$-dimensional Riemannian space, where $p \geq 2$ is the number of control axes. The vector representation of the equation is

$\mathbf{r}=\mathbf{r}(u), u \in\left[u_{0}, u_{E}\right]$,

where $u_{0}$ is the value of parameter at time $t=t_{0}$;

$U_{E}$ is a parameter value at the moment of time $t=t_{E}$;

$t$ is a current time, s.

For example, the parametric trajectory equation for threeaxis machining with programmable axes $X, Y, Z$ are:

$X=x(u), Y=(u), Z=z(u)$.

The differential motion characteristics of velocity, acceleration and jerk are defined as the corresponding derivatives of expression (1) with respect to time:

$\mathbf{v}=\frac{\mathrm{d} \mathbf{r}}{\mathrm{d} t} ;$

$\mathbf{a}=\frac{\mathrm{d} \mathbf{v}}{\mathrm{d} t}=\frac{\mathrm{d}^{2} \mathbf{r}}{\mathrm{d} t^{2}}$

$\mathbf{j}=\frac{\mathrm{d} \mathbf{a}}{\mathrm{d} t}=\frac{\mathrm{d}^{2} \mathbf{v}}{\mathrm{d} t^{2}}=\frac{\mathrm{d}^{3} \mathbf{r}}{\mathrm{d} t^{3}}$.

It is known that to ensure smoothness of the jerk, it is necessary to provide the C2-continuity of function (5). Respectively, application of at least the fifth-order spline to represent the trajectory (1) can ensure a jerk smoothness.

At the motion along a curved toolpath, the vectors of the velocity, acceleration and jerk are decomposed to coordinate axes for computing the values of kinematic parameters for each drive. In accordance with the smoothness hypothesis, the movement of each individual axis should be performed with a smooth change in the differential characteristics up to a jerk. At the same time, the smoothness of the kinematic parameters change for each axis depends not only on the smoothness of the path (1), but also on the smoothness of change in the feed along the path.

As discussed earlier, other researchers have proposed various algorithms for S-shape feedrate scheduling, but Sshape with the smooth jerk has not been investigated. In this paper, a mathematical model for S-shape feedrate profile with smoothly-limited jerk based on the use of trigonometric function $\sin ^{2}$ is proposed. 


\section{MATHEMATICAL MODEL OF FEEDRATE PROFILE WITH SMOOTHLY-LIMITED JERK}

Forming of smooth laws for velocity changing during acceleration or deceleration process is one of the tasks, the solution of which contributes to the smooth movement of each axis drive of a CNC machine. In this paper, considered only the key issues of S-shape formation related to the subsystem "Feed generation $\mathrm{V}(\mathrm{t})$ " (Fig. 1). The issues of ensuring the functioning of the subsystem "Velocity planning $\mathrm{Vc}(\mathrm{u})$ " are not considered, since they do not reflect the main idea of this work on the effect of the jerk smoothness on the accuracy of trajectory movements.

To achieve this goal, the authors of this article propose to use the $\sin ^{2}$ function to form the feed change profile in the acceleration-deceleration process. [Sosonkin 2005] mentioned a possible use of the $\sin ^{2}$ function to form the feedrate or acceleration changing laws, but explainations of this solution had not been submitted. The following symbols for kinematic processing parameters are proposed:

$F_{0}$ is a feedrate at the moment of frame beginning, $\mathrm{m} / \mathrm{s}$;

$F$ is a specified feedrate, $\mathrm{m} / \mathrm{s}$;

$A_{P}$ is a largest achieved acceleration, $\mathrm{m} / \mathrm{s}^{2}$;

$A_{\max }$ is a maximum acceleration limit, $\mathrm{m} / \mathrm{s}^{2}$;

$J_{P}$ is a largest achieved jerk $\mathrm{m} / \mathrm{s}^{3}$;

$J_{\max }$ is a maximum jerk limit, $\mathrm{m} / \mathrm{s}^{3}$;

$D J_{\max }$ is a maximum jerk derivative (snap) limit, $\mathrm{m} / \mathrm{s}^{4}$;

$\Delta t_{P}$ is a duration of velocity change from $F_{0}$ to $F, \mathrm{~s}$;

$t_{P}$ is an end time for velocity change, $\mathrm{s}$;

$S(t)$ is a travel function, $\mathrm{m}$;

$V(t)$ is a velocity along the trajectory (feedrate), $\mathrm{m} / \mathrm{s}$;

$A(t)$ is a acceleration function, $\mathrm{m} / \mathrm{s}^{2}$;

$J(t)$ is a jerk function, $\mathrm{m} / \mathrm{s}^{3}$;

$D J(t)$ is a jerk derivative (snap) function, $\mathrm{m} / \mathrm{s}^{4}$.

The following boundary conditions should be observed to achieve the smoothness of tool motion during acceleration: $V\left(t_{0}\right)=F_{0} ; \quad V\left(t_{P}\right)=F ; \quad A\left(t_{0}\right)=A\left(t_{P}\right)=0 ; \quad J\left(t_{0}\right)=J\left(t_{P}\right)=0 ;$ $D J\left(t_{0}\right)=D J\left(t_{P}\right)=0$.

The S-shape feedrate profile (Fig. 3), which usually includes seven intervals, is proposed:

$T_{i}=\left[t_{i-1}, t_{i}\right], i \in[1,7]$.

Acceleration starts at time $t_{0}$ and fininishes at $t_{7}=t_{P}$, complete process duration is $\Delta t_{P}$. The model covers the following intervals:

$T_{1}$ is a jerk change from $J\left(t_{0}\right)=0$ to $J\left(t_{1}\right)=J_{P}$, interval duration is $\Delta t_{1}$

$T_{2}$ is an acceleration change with constant jerk $J\left(t_{1} \ldots t_{2}\right)=J_{P}=$ const, interval duration $\Delta t_{2}$;

$T_{3}$ is a jerk change from $J\left(t_{2}\right)=J_{P}$ to $J\left(t_{3}\right)=0$ and achievement of the largest acceleration $A\left(t_{3}\right)=A p$;

$T_{4}$ is a motion with constant acceleration $A\left(t_{3} \ldots t_{4}\right)=$ const, interval duration $\Delta t 4$;

$T_{5}$ is a jerk change from $J\left(t_{4}\right)=0$ to $J\left(t_{5}\right)=-J_{P}$;

$T_{6}$ is an acceleration change with constant jerk $J\left(t_{5} \ldots t_{6}\right)=-J P$

$T_{7}$ is a jerk change from $J\left(t_{6}\right)=-J_{P}$ to $J\left(t_{P}\right)=0$ and acceleration decreasing to $A\left(t_{P}\right)=0$.
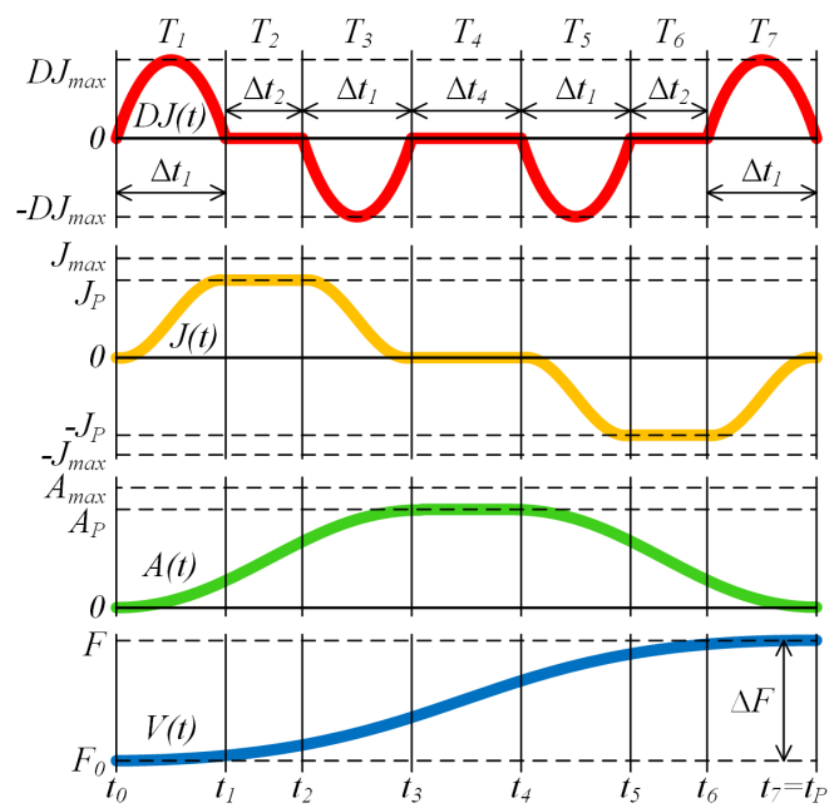

Fig. 3: The proposed profile with smoothly-limited jerk.

In the particular case, intervals of constant acceleration $T_{4}$ and/or constant jerk $T_{2}, T_{6}$ may be not used.

At each $i$ interval time from it start is defined as

$\tau(t)=\left(t-t_{i-1}\right)$, where $t \in T_{i}$.

Complete process time is

$\Delta t_{P}=t_{7}-t_{0}$.

The jerk changing law is based on the $\sin ^{2}$ function with period defined by angular frequency $\omega\left(\mathrm{s}^{-1}\right)$. Value of $\omega$ is computed from the condition $\sin ^{2}\left(\omega \cdot \Delta t_{1}\right)=1$ :

$\omega=\pi /\left(2 \Delta t_{1}\right)$.

The largest achieved jerk of process $J_{P}$ must not be more than the $J_{\max }$ limit:

$J_{P} \leq J_{\max }$.

The jerk changing function is defined as:

$J(t)= \begin{cases}J_{\mathrm{P}} \cdot F_{J 1}(t) & t \in T_{1} \\ J_{\mathrm{P}} & t \in T_{2} \\ J_{\mathrm{P}} \cdot F_{J 2}(t) & t \in T_{3} \\ 0 & t \in T_{4} \\ -J_{\mathrm{P}} \cdot F_{J 1}(t) & t \in T_{5} \\ -J_{\mathrm{P}} & t \in T_{6} \\ -J_{\mathrm{P}} \cdot F_{J 2}(t) & t \in T_{7}\end{cases}$

where

$$
F_{J 1}(t)=\sin ^{2}[\omega \cdot \tau(t)]
$$

$F_{J 2}(t)=\sin ^{2}[\pi / 2+\omega \cdot \tau(t)]$.

The jerk derivative function $D J(t)$ is found by differentiation of the system (11): 


$$
D J(t)= \begin{cases}J_{\mathrm{P}} \cdot F_{D J 1}(t) & t \in T_{1} \\ 0 & t \in T_{2} \\ J_{\mathrm{P}} \cdot F_{D J 2}(t) & t \in T_{3} \\ 0 & t \in T_{4}, \\ -J_{\mathrm{P}} \cdot F_{D J 1}(t) & t \in T_{5} \\ 0 & t \in T_{6} \\ -J_{\mathrm{P}} \cdot F_{D J 2}(t) & t \in T_{7}\end{cases}
$$

where

$$
F_{D J 1}(t)=\omega \cdot \sin [2 \omega \tau(t)] ; F_{D J 2}(t)=\omega \cdot \sin [\pi+2 \omega \tau(t)] ;
$$

Acceleration, velocity and traveled distance functions can be obtained by sequential integration of jerk, acceleration and velocity functions (Fig. 3):

$$
A(t)= \begin{cases}J_{\mathrm{P}} \cdot F_{A 1}(t)+A_{0}(t) & t \in T_{1} \\ J_{\mathrm{P}} \cdot \tau(t)+A_{1}(t) & t \in T_{2} \\ J_{\mathrm{P}} \cdot F_{A 2}(t)+A_{2}(t) & t \in T_{3} \\ A_{3}(t) & t \in T_{4}, \\ -J_{\mathrm{P}} \cdot F_{A 1}(t)+A_{4}(t) & t \in T_{5} \\ -J_{\mathrm{P}} \cdot \tau(t)+A_{5}(t) & t \in T_{6} \\ -J_{\mathrm{P}} \cdot F_{A 2}(t)+A_{6}(t) & t \in T_{7}\end{cases}
$$$$
A(t)=\int J(t) d t ; V(t)=\int A(t) d t ; S(t)=\int V(t) d t .
$$

where: $A_{i}(t)=A\left(t_{i}\right)$;

$$
\begin{gathered}
F_{A 1}(t)=\frac{1}{2} \tau(t)-\frac{1}{4 \omega} \sin [2 \omega \cdot \tau(t)] ; \\
F_{A 2}(t)=\frac{1}{2} \tau(t)+\frac{1}{4 \omega} \sin [2 \omega \cdot \tau(t)] . \\
V(t)= \begin{cases}J_{\mathrm{P}} \cdot F_{V 1}(t)+V_{0}(t) & t \in T_{1} \\
J_{\mathrm{P}} \cdot \frac{1}{2} \tau^{2}(t)+V_{1}(t) & t \in T_{2} \\
J_{\mathrm{P}} \cdot F_{V 2}(t)+V_{2}(t) & t \in T_{3} \\
V_{3}(t) & t \in T_{4}, \\
-J_{\mathrm{P}} \cdot F_{V 1}(t)+V_{4}(t) & t \in T_{5} \\
-J_{\mathrm{P}} \cdot \frac{1}{2} \tau^{2}(t)+V_{5}(t) & t \in T_{6} \\
-J_{\mathrm{P}} \cdot F_{V 2}(t)+V_{6}(t) & t \in T_{7}\end{cases}
\end{gathered}
$$

where: $V_{i}(t)=V\left(t_{i}\right)+A_{i}(t) \cdot \tau(t)$;

$$
\begin{aligned}
& F_{V 1}(t)=\frac{1}{2} \tau^{2}(t)-\frac{1}{4 \omega^{2}} \sin ^{2}[\omega \cdot \tau(t)] ; \\
& F_{V 2}(t)=\frac{1}{2} \tau^{2}(t)+\frac{1}{4 \omega^{2}} \sin ^{2}[\omega \cdot \tau(t)] . \\
& S(t)= \begin{cases}J_{\mathrm{P}} \cdot F_{S 1}(t)+S_{0}(t) & t \in T_{1} \\
J_{\mathrm{P}} \cdot \frac{1}{6} \tau^{3}(t)+S_{1}(t) & t \in T_{2} \\
J_{\mathrm{P}} \cdot F_{S 2}(t)+S_{2}(t) & t \in T_{3} \\
S_{3}(t) & t \in T_{4}, \\
-J_{\mathrm{P}} \cdot F_{S 1}(t)+S_{4}(t) & t \in T_{5} \\
-J_{\mathrm{P}} \cdot \frac{1}{6} \tau^{3}(t)+S_{5}(t) & t \in T_{6} \\
-J_{\mathrm{P}} \cdot F_{S 2}(t)+S_{6}(t) & t \in T_{7}\end{cases}
\end{aligned}
$$

where: $S_{i}(t)=S\left(t_{i}\right)+V\left(t_{i}\right) \cdot \tau(t)+\frac{1}{2} A\left(t_{i}\right) \tau^{2}(t)$;

$$
\begin{aligned}
& F_{S 1}(t)=\frac{1}{12} \tau^{3}(t)-\frac{1}{8 \omega^{2}} \tau(t)+\frac{1}{16 \omega^{3}} \sin [2 \omega \cdot \tau(t)] \\
& F_{S 2}(t)=\frac{1}{12} \tau^{3}(t)+\frac{1}{8 \omega^{2}} \tau(t)-\frac{1}{16 \omega^{3}} \sin [2 \omega \cdot \tau(t)] .
\end{aligned}
$$

It should be noted that the largest process acceleration $A_{P}$ achieved at time moment $t_{3}$ should not be more than $A_{\max }$

$A_{P} \leq A_{\max }$.

Complete acceleration time for the proposed model is

$\Delta t_{P}=4 \Delta t_{1}+2 \Delta t_{2}+\Delta t_{4}$.

It is needed to obtain the equations for computatin of kinematic parameters on boundaries of several intervals of process in moments $t_{0} \ldots t_{7}$. The jerk, acceleration, velocity and traveled distance, which will be achieved at the end of $T_{1}$ interval (at the $\left.t_{1}\right)$, are found from $(11)-(15)$ :

$$
\begin{aligned}
& J\left(t_{1}\right)=J_{P} ; \\
& A\left(t_{1}\right)=J_{P} \cdot \frac{1}{2} \Delta t_{1} ; \\
& V\left(t_{1}\right)=J_{P} \cdot\left(\frac{1}{4}-\frac{1}{\pi^{2}}\right) \cdot \Delta t_{1}^{2}+F_{0} ; \\
& S\left(t_{1}\right)=J_{P} \cdot\left(\frac{1}{12}-\frac{1}{2 \pi^{2}}\right) \cdot \Delta t_{1}^{3}+F_{0} \cdot \Delta t_{1} .
\end{aligned}
$$

Maximum jerk derivative value will be achieved at interval $T_{1}$ and is defined from the equation (12):

$$
D J_{\max }=J_{P} \omega=J_{P} \cdot \frac{\pi}{2 \Delta t_{1}} .
$$

The largest process acceleration will be achieved at interval $T_{3}$ and is defined from the equation (13) considering (16):

$A_{P}=A\left(t_{3}\right)=J_{P} \cdot\left(\Delta t_{1}+\Delta t_{2}\right)$.

Velocity achieved at the end of $T_{7}$ interval is found from the equation (14):

$$
\begin{aligned}
F=V\left(t_{7}\right)=J_{P} & \cdot\left(2 \Delta t_{1}^{2}+3 \Delta t_{1} \Delta t_{2}+\Delta t_{2}^{2}\right)+ \\
& +J_{P} \cdot\left(\Delta t_{1}+\Delta t_{2}\right) \cdot \Delta t_{4}+F_{0}
\end{aligned}
$$

Thus, a mathematical model of velocity changing along a trajectory using the S-curve acceleration with smoothlylimited jerk is created. This model ensures a smooth change of velocity, acceleration and jerk, as well as continuity and limitation of the snap change. Equations for deceleration along trajectory are formed similarly.

\section{DETERMINATION OF TIME INTERVALS FOR ACCELERATION TAKING INTO ACCOUNT KINEMATIC PARAMETERS LIMITS}

Equipment features, such as $A_{\max }, J_{\max }, D J_{\max }$ and velocity change value $\left(F-F_{0}\right)$, significantly influence the structure of the generated law of acceleration. At a certain combination of parameters intervals $T_{2}, T_{4}, T_{6}$ can be absent. The main cases and equations for determinaion of the velocity changing parameters are considered.

Time interval $\left.\Delta t_{1}\right\lrcorner$ needed to achieve the maximum jerk (Fig. $4 a)$ is determined from equation (22)

$$
\Delta t_{1 J}=\frac{\pi \cdot J_{\max }}{2 D J_{\max }} .
$$



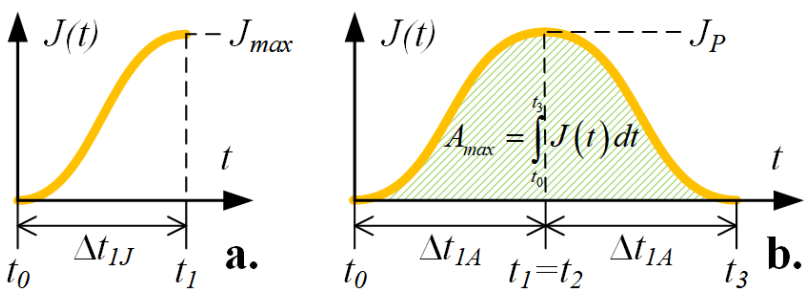

Fig. 4: Determination of $\Delta t_{1}$ at the conditions of achievement $J_{\max }$ or $A_{\max }$

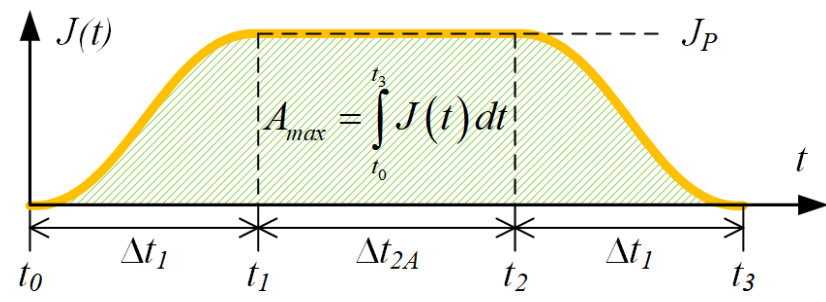

Fig. 5: Determination of $\Delta t_{2}$ at the condition of achievement $A_{\max }$

The maximum acceleration can be achieved, when acceleration is carried along a shortened (Fig. 4b) or full (Fig. 5) jerk change diagram.

There is no interval $\mathrm{T}_{2}$ in the shortened diagram and $\Delta t_{2}=0$. In this case, the duration of the initial acceleration interval $\Delta t_{1} \mathrm{~A}$ required to achieve the maximum acceleration value is determined from the expressions (22) and (23)

$\Delta \mathrm{t}_{1 \mathrm{~A}}=\sqrt{\frac{\pi A_{\max }}{2 D J_{\max }}}$.

The time $\Delta t_{2 A}$ required to achieve the maximum acceleration in the full diagram (Fig. 5) is determined from the expressions (22) and (23)

$\Delta \mathrm{t}_{2 \mathrm{~A}}=\frac{A_{\max }}{J_{P}}-\frac{\pi J_{P}}{2 D J_{\max }}$.

The specified velocity change can be achieved, when acceleration is carried along one of two shortened (Fig. 6, 7) or full (Fig. 1) diagrams.

When velocity changes $\Delta F$ are small, the intervals $T_{2}, T_{4}, T_{6}$ (Fig. 6) may be not used. For this case $\Delta t_{2}=\Delta t_{4}=0$ and according to equation (17) full process time is determined by duration $T_{1}$

$t_{P}=4 \Delta t_{1 F}$.

For this case the expression for $\Delta t_{1} F$, which ensures achievement of the specified velocity change value, can be obtained from the (22) and (24) equations

$\Delta t_{1 F}=\sqrt[3]{\frac{\pi \cdot \Delta F}{4 \cdot D J_{\max }}}$.

For the case, when a given velocity change can be achieved according to the acceleration diagram without an interval $T_{4}$ (Fig. 7), the $\Delta t_{2 F}$ duration is determined from equations (22) and (24)

$$
\Delta t_{2 F}=\sqrt{\frac{J_{P}^{2} \cdot \pi^{2}}{16 D J_{\max }^{2}}+\frac{\Delta F}{J_{P}}}-\frac{3 J_{P} \pi}{4 D J_{\max }} .
$$

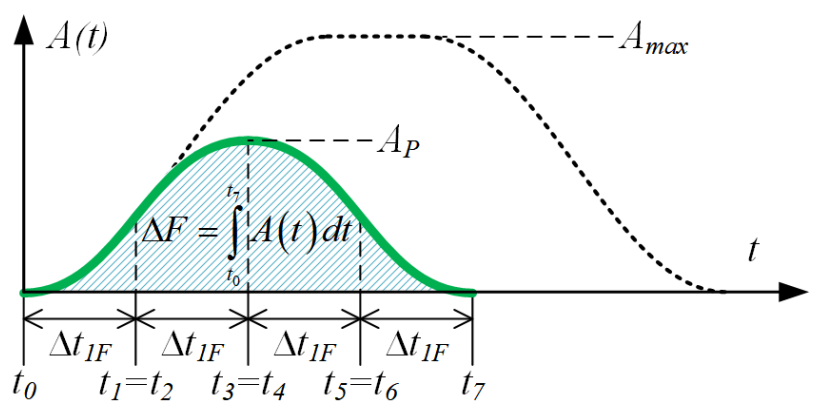

Fig. 6: Determination of $\Delta t_{1}$ at the condition of achievement $\Delta F$.

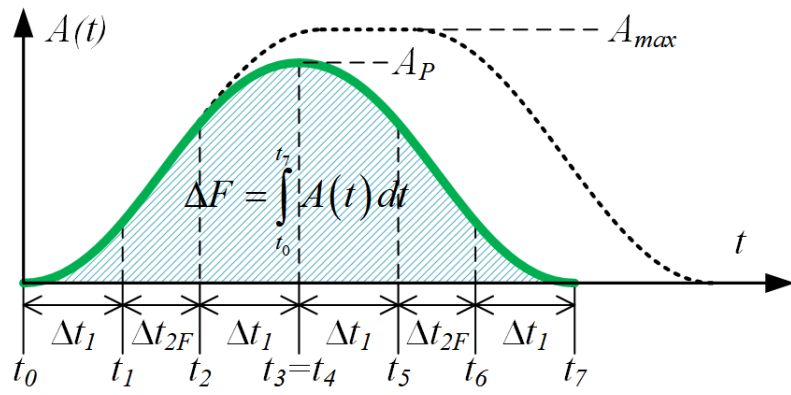

Fig. 7: Determination of $\Delta t_{2}$ at the condition of achievement $\Delta F$.

When implementing a full acceleration diagram, the duration of interval $T_{4}$ is determined from (22), (23), (24), (27)

$\Delta t_{4 F}=\frac{\Delta F}{A_{P}}-\frac{\pi J_{P}}{2 D J_{\max }}+\frac{A_{P}}{J_{P}}$

The expression for determining the jerk achieved at the velocity changing process is obtained from equation (22)

$J_{P}=\frac{2}{\pi} \Delta t_{1} D J_{\max }$.

It should be noted that the durations of acceleration intervals $\Delta t_{i}$ cannot be arbitrary, but must satisfy the condition of control cycle duration of a CNC system

$\Delta t_{i}=m \cdot \Delta \tau$,

where $\Delta \tau$ is a duration of one CNC time cycle (for example, $0.001 \mathrm{~s}) ; m$ is a number of cycles (positive integer).

The rounding off the durations of intervals in accordance with the expression (33) leads to the error of setting the final acceleration-deceleration rate, when calculated from the expression (14). To eliminate these errors, the value of the jerk $J_{P}$ must be adjusted. The expression for the jerk, which provides an accurate calculation of the velocity change, is obtained from the (24) equation

$$
J_{P}=\frac{F-F_{0}}{2 \cdot \Delta t_{1}^{2}+3 \cdot \Delta t_{1} \cdot \Delta t_{2}+\Delta t_{2}^{2}+\Delta t_{4} \cdot\left(\Delta t_{1}+\Delta t_{2}\right)} .
$$

Tthe algorithm for determining the parameters of velocity changing process is considered as the following. At the first stage $\Delta t_{1}$ (25), $\Delta t_{1 A}(26), \Delta t_{1 F}(29)$ are calculated. The smallest of them is selected as the $\Delta t_{1}$ time interval

$\Delta t_{1}=\min \left(\Delta t_{1 J}, \Delta t_{1 A}, \Delta t_{1 F}\right)$. 
For the obtained $\Delta t_{1}$ time interval the value of the largest attainable jerk $J_{P}$ is determined from the expression (32), which is further used in the calculation of $\Delta t_{2 A}(27), \Delta t_{2 F}(30)$. The smallest of them is chosen as the $\Delta t_{2}$ time interval. If a negative value is obtained, then $\Delta t_{2}$ is assumed to be zero

$\Delta t_{2}=\max \left[\min \left(\Delta t_{2 A}, \Delta t_{2 F}\right), 0\right]$.

Using obtained values $J_{P}(32), \Delta t_{1}(35)$ and $\Delta t_{2}(36)$, the $A_{P}$ value is calculated from (23).

Value of $\Delta t_{4 F}(31)$ is calculated. Duration of $T_{4}$ is defined as:

$\Delta t_{4}=\max \left(\Delta t_{4 F}, 0\right)$.

Using calculated values $\Delta t_{1}, \Delta t_{2}, \Delta t_{4}$, the boundaries of intervals $t_{i}$ are determined. The $J_{P}$ jerk value, which provides an accurate calculation of the velocity change, is computed from the (34) equation.

\section{EXPERIMENTAL INVESTIGATIONS}

To confirm the validity of the presented model for S-shape feedrate profile, confirm the validity of the smoothness hypothesis and the possibility of computation of tool movement velocity during acceleration-deceleration, several results of numerical experiments and experiments on a CNC machine are submitted.

\subsection{Numerical experiments}

The possibility of forming an S-shape feedrate profile with a different structure is demonstrated in numerical experiments. The presented model allows to form different types of velocity change curves depending on the preset values of the limits $A_{\max }, J_{\max }, D J_{\max }$ and velocity change value $\Delta F$ during acceleration-deceleration. Table 1 shows examples illustrating this possibility.

For Test 1 the limits are specified in such a manner that trapezoidal acceleration profile with limited jerk is formed. This is achieved by setting a high value of $D J_{\max }$. For Test 2 the parameters are set to ensure the formation of the $S$ shape feedrate profile with smoothly-limited jerk. Naturally, with the same limitations of jerk $\left(16 \mathrm{~m} / \mathrm{s}^{3}\right)$ and acceleration $\left(1.57 \mathrm{~m} / \mathrm{s}^{2}\right)$ and the same given velocity change value $(13.2 \mathrm{~m} / \mathrm{min})$ Test 2 provides acceleration of $0.046 \mathrm{~s}$ longer, when compared with Test 1 . In this example, the increase in acceleration time is $16 \%$ the duration of acceleration process.

Tests 3-5 shows special cases of the formation of the Sshape feedrate profile, for which there are no intervals $T_{2}$, $T_{4}, T_{6}$. In Test 3, the S-shape feedrate profile was formed, in which there was no $T_{4}$ interval, and the acceleration $A_{P}$ was less than the maximum permitted acceleration $A_{\max }$. Test 4 shows that for small $\Delta F$ values, acceleration is performed with the S-shape feedrate profile, in which neither $J_{P}$ nor $A_{P}$ reaches their maximum values. In this case, there are no intervals $T_{2}, T_{4}, T_{6}$.

Tab. 1: S-shape feedrate profile computation results.

\begin{tabular}{lccccc}
\hline Parameter & Test $\mathbf{1}$ & Test 2 & Test 3 & Test 4 & Test 5 \\
\hline$\Delta F, \mathrm{~m} / \mathrm{min}$ & 13.2 & 13.2 & 4.8 & 0.9 & 9.0 \\
$\Delta F, \mathrm{~m} / \mathrm{s}$ & 0.22 & 0.22 & 0.08 & 0.015 & 0.15 \\
$A_{\max }, \mathrm{m} / \mathrm{s}^{2}$ & 1.57 & 1.57 & 1.57 & 1.57 & 1.05 \\
$J_{\max }, \mathrm{m} / \mathrm{s}^{3}$ & 16 & 16 & 16 & 16 & 24 \\
$D J_{\max }, \mathrm{m} / \mathrm{s}^{4}$ & 25132 & 560 & 560 & 560 & 500 \\
$\Delta t_{1} \mathrm{~J}, \mathrm{~s}$ & 0.001 & 0.044 & 0.044 & 0.044 & 0.075
\end{tabular}

\begin{tabular}{lccccc}
$\Delta t_{1} A, \mathrm{~s}$ & 0.009 & 0.066 & 0.066 & 0.066 & 0.057 \\
$\Delta t_{1}, \mathrm{~s}$ & 0.019 & 0.067 & 0.048 & 0.027 & 0.061 \\
$\Delta t_{1}, \mathrm{~s}$ & 0.001 & 0.044 & 0.044 & 0.027 & 0.057 \\
$J_{P}, \mathrm{~m} / \mathrm{s}^{3}$ & 16.000 & 15.686 & 15.686 & 6.685 & 18.144 \\
$\Delta t_{2 A}, \mathrm{~s}$ & 0.097 & 0.056 & 0.056 & 0.136 & 0 \\
$\Delta t_{2 F}, \mathrm{~s}$ & 0.116 & 0.054 & 0.009 & 0.001 & 0.01 \\
$\Delta t_{2}, \mathrm{~s}$ & 0.097 & 0.054 & 0.009 & 0.001 & 0 \\
$A_{P}, \mathrm{~m} / \mathrm{s}^{2}$ & 1.568 & 1.537 & 0.831 & 0.270 & 1.034 \\
$\Delta t_{4 F}, \mathrm{~s}$ & 0.041 & 0.001 & -0.001 & 0.001 & 0.031 \\
$\Delta t_{4}, \mathrm{~s}$ & 0.041 & 0.001 & 0 & 0.001 & 0.031 \\
$\Delta t_{P}, \mathrm{~s}$ & 0.239 & 0.285 & 0.194 & 0.111 & 0.259 \\
$J_{P}, \mathrm{~m} / \mathrm{s}^{3}$ & 16.035 & 15.699 & 15.561 & 9.566 & 18.149 \\
$F, \mathrm{~m} / \mathrm{s}$ & 0.22 & 0.22 & 0.08 & 0.015 & 0.15 \\
\hline
\end{tabular}

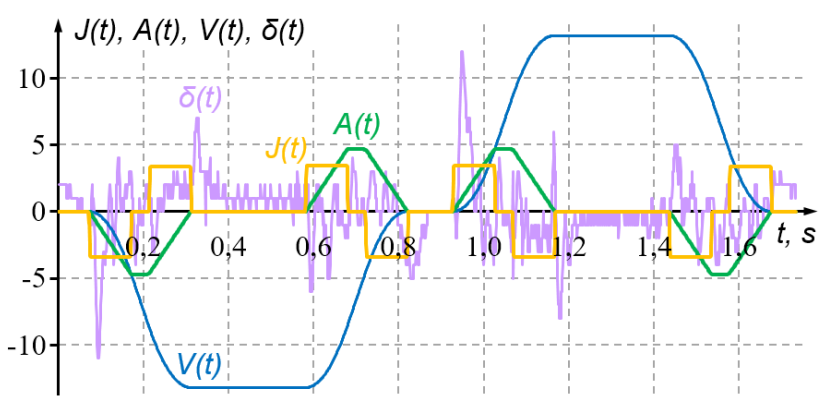

Fig. 8: Servo errors during point-to-point motion with using trapezoidal acceleration profile with jerk limited.

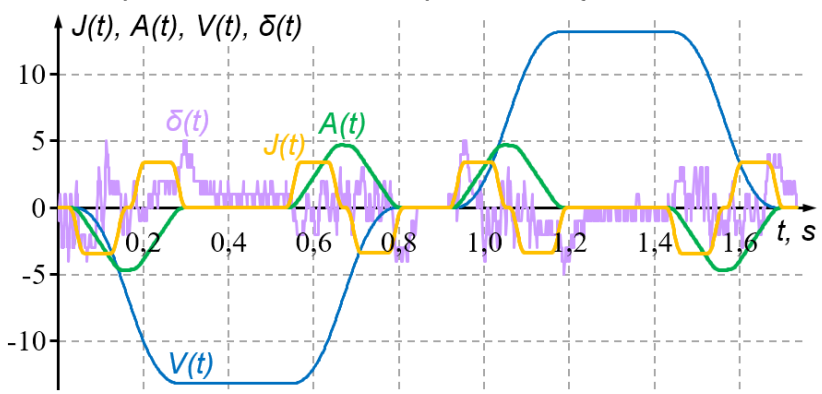

Fig. 9: Servo errors during point-to-point motion with using S-shape feedrate profile with smoothly-limited jer.k.

Test 5 shows that for large $\Delta F$ values and uncoordinated $A_{\max }$ and $J_{\max }$ values, the $\mathrm{S}$-shape feedrate profile is formed, in which $T_{2}, T_{6}$ intervals do not exist and $T_{4}$ interval of motion at maximum acceleration is present. For such a combination of parameters the $J_{\max }$ limit value will not be reached at any velocity changes $\Delta F$.

Thus, the universality of the proposed model is shown and the change in the structure of the S-shape feedrate profile for various initial conditions is demonstrated.

\subsection{Experiments on a CNC machine}

In order to confirm the validity of the smoothness hypothesis, an experimental study was conducted on a CNC machine. Testing is focused on identifying the influence of the smoothness of the S-shape formed in "Feed generation $\mathrm{V}(\mathrm{t})$ )" (Fig. 1) on the behavior of high-speed equipment. According to this approach, the most correct test is the point-to-point motion test. This approach eliminates the influence of related factors: velocity planning errors along a curved path (occurs in the subsystem "Velocity planning $\mathrm{Vc}(\mathrm{u})$ )"); errors of interpolation of a curved path (in "Trajectory interpolation"); features of the regulator (in "Position regulator").

The movement of the machine axis with $A C$ servomotor Estun EMG-10APA22 was investigated. The experiment investigated the behavior of servo errors $\delta(t)$ during point- 
to-point motion. The axis motion was compared when applying the acceleration profiles trapezoidal acceleration profile with jerk limited (Table 1, Test 1) and S-shape feedrate profile with smoothly-limited jerk (Table 1, Test 2). The resulting Servo errors $\delta(t)$ for Test 1 (Fig. 8) are two times over than servo errors $\delta(t)$ for Test 2 (Fig. 9). The results of this experiment confirm that the use of the $S$ shape feedrate profile with smoothly-limited jerk contributes to improvement of motion accuracy of machine organs compared to the use of S-shape feedrate profile with trapezoidal acceleration and jerk limited. This result confirms the formulated hypothesis of smoothness.

\section{SUMMARY}

The hypothesis of the kinematic motion parameters smoothness is formulated: if any differential characteristic has an influence on the motion process, then it should be limited and change smoothly.

Algorithm of velocity changing along trajectory using the Scurve acceleration-deceleration with smoothly-limited jerk is proposed. The S-shape feedrate profile with smoothlylimited jerk model based on application of trigonometric function $\sin ^{2}$ is proposed.

A distinctive feature of the algorithm is to ensure a smooth change of not only the speed and acceleration of movement along the trajectory, but also the smoothness of the jerk and snap continuity taking into account their maximum permissible values.

The presented model, depending on the set values of limits and the velocity change value in the accelerationdeceleration process, allows the formation of various types of feed variation curves. Using of the model for forming of trapezoidal acceleration profile with jerk limited and the Sshape feedrate profile with smoothly-limited jerk is demonstrated. The results of numerical experiments showed the universality of the proposed model.

Experiments on the CNC machine showed that the use of Sshape feedrate profile with smoothly-limited jerk contributes to an increase in the accuracy of movement of machine organs compared to S-shape feedrate profile with trapezoidal acceleration and jerk limited. This result confirms the smoothness hypothesis formulated in this article.

\section{REFERENCES}

[Beudaert 2012] Beudaert, X., Lavernhe, S. and Tournier, C. Feedrate interpolation with axis jerk constraints on 5-axis NURBS and G1 tool path. International Journal of Machine Tools \& Manufacture, 2012, Vol.57, pp.73-82. ISSN: 08906955

[Boerlage 2004] Boerlage, M., Tousain, R. and Steinbuch M. Jerk derivative feedforward control for motion systems. Proceedings of the American Control Conference, Boston Sheraton Hotel, Boston, MA, USA, 30 June-2 July, 2004 Vol.5, Evanston: Northwestern Univercity, pp.4843-4848. ISBN: 0-7803-8335-4

[Erkorkmaz 2001] Erkorkmaz, K. and Altintas, Y. High speed CNC system design. Part I: Jerk limited trajectory generation and quintic spline interpolation. International Journal of Machine Tools and Manufacture, 2001, Vol.41(9), pp.1323-1345. ISSN: 0890-6955

[Fan 2012] Fan, W., Gao, X.-S., Yan, W. and Yuan, C.-M. Interpolation of parametric CNC machining path under confined jounce. The International Journal of Advanced Manufacturing Technology, 2012, Vol.62, No.5-8, pp.719739. ISSN 0268-3768

[Krivtsov 2012] Krivtsov, V.S., Kombarov, V.V. and Sorokin, V.F. Problems of spline interpolation with smoothly-limited kinematical parameters of movement in tasks of CNC highspeed equipment. Aerospace Engineering and Technology, 2012, Vol.9(96), pp.11-19. ISSN: 1727-7337

[Lartigue 2001] Lartigue, C., Thiebaut, F., Maekawa, T. CNC tool path in terms of B-spline curves. CAD Computer Aided Design, 2001, Vol.33(4), p. 307-319

[Lee 2011] Lee, A.-C Lin, M.-T., Pan, Y.-R. and Lin, W.Y.The feedrate scheduling of NURBS interpolator for CNC machine tools. CAD Computer Aided Design, 2011, Vol.43(6), pp.612-628. ISSN: 0010-4485

[Liu 2016] Liu, Q., Liu, H. and Yuan, S. High accurate interpolation of NURBS tool path for CNC machine tools Chin. J. Mechanical Engineering, 2016, Vol.29, No.5, pp.911-920. ISSN: 2187-9745

[Ni 2018] Ni, H., Yuan, J., Ji, S., Zhang, C. and Hu, T. Feedrate scheduling of NURBS interpolation based on a novel jerk-continuous ACC/DEC Algorithm. IEEE Access, 2018, Vol.6, pp.66403-66417. ISSN: 2169-3536

[Novikova 2012] Novikova, E.A. Substantion of criteria of smoothness of mechatronic drives of forward moving. Fundamental research, 2012, Vol.3, pp.123-128. ISSN: $1812-7339$

[Petrakov 2017] Petrakov, Y., Danylchenko, M., Petryshyn, A. Programming spindle speed variation in turning. EasternEuropean Journal of Enterprise Technologies, 2017, Vol. 2(1-85), pp.4-9. ISSN: 1729-3774

[Prévost 2011] Prévost, D., Lavernhe, S., Lartigue, Cl. and Dumur, D. Feed drive modelling for the simulation of tool path tracking in multi-axis High Speed Machining. International Journal of Mechatronics and Manufacturing Systems, 2011, Vol.4, Iss.3/4, pp. 266-284. ISSN: 17531047.

[Sorokin 2002] Sorokin, V.F. Mathematical model of complicated shaped surface for adaptive programmed control of metalworking equipment. Technological systems, 2002. Vol.5(16), p.44-51. ISSN: 2074-0603.

[Sorokin 2012] Sorokin, V.F. and Kombarov, V.V. Comparison of kinematical parameters of movement at path modeling of high-speed CNC processing with cubic and quantic splines Aerospace Engineering and Technology, 2012, Vol.8(95), pp.11-17. ISSN: 1727-7337

[Sosonkin 2005] Sosonkin, V.L. and Martinov G.M. Computer numerical control systems, 2005, 296 p ISBN: 598704-012-4.

[Zhang 2017] Zhang, W., Gao, S., Cheng, X. and Zhang, F. An innovation on high-grade CNC machines tools for $B$ spline curve method of high-speed interpolation arithmetic AIP Conference Proceedings, 2017, 1834,040013. 